\title{
Duns Scot et le Liber de causis
}

\author{
Jean-Michel Counet \\ Université catholique de Louvain
}

Disons-le d'emblée: les références au Liber de causis chez Dus Scot sont assez rares et lorsqu' il est fait mention dans l' œuvre du Docteur Subtil d' une proposition du Liber, le spectre des propositions concernées est très limité.

À ma connaissance, Duns Scot ne fait aucune référence à certaines propositions qui jouent un rôle central chez d' autres auteurs, je pense par exemple à la proposition $\mathrm{Xx}(\mathrm{XXI})$ selon laquelle la cause première est riche par soi $^{1}$ et à la célèbre thèse $\mathrm{XIV}(\mathrm{XV})$ que tout être qui connaît sa propre essence fait retour vers elle d'un retour total ${ }^{2}$.

D' autres propositions fondamentales pour d' autres auteurs, comme la proposition IV, «l' être est la première des choses créées ${ }^{3}$, ou le développement explicatif qui clôt le chapitre $\operatorname{IX}(x)$, «tout ce qui est reçu est reçu sous le mode du récipient», sont certes présentes chez Duns Scot, mais en très peu d'occurrences. Pour considérer ce dernier cas, l' axiome: «tout ce qui est reçu l'est sur le mode du récipient» n' est mentionnée, à ma connaissance, que dans son Commentaire à l'Isagogè de Porphyre ${ }^{4}$, pour asseoir la thèse qu' un accident commun reçu dans une substance singulière individuelle ne peut être lui-même qu' individuel.

Dans le même sens, la proposition importante du De causis comme quoi, entre les êtres dont la substance et l'activité sont mesurées par l'éternité et les êtresdont la substance et l'activité sont mesurées par le temps, il y a des êtres dont la substance est mesurée par l'éternité et l' activité mesurée par le temps, n' est mentionnée qu'une fois, dans une discussion sur laquelle je reviendrai. Bien entendu, le fait qu' il n'y ait pas ou qu'il y ait très peu de références à telle ou telle proposition n'implique nullement que les problématiques visées par

1 Liber de causis, p. 92; trad. française P. Magnard et alii, p. 7o.

2 Liber de causis, p. 79; Magnard et alii, p. 63.

3 Liber de causis, p. 54; Magnard et alii, p. 45; Jean Duns Scot, Ordinatio I, d. 3, q. 4, n 208, p. 126. Duns Scot invoque cette proposition en faveur d'une thèse d'Henri De Gand, selon laquelle le concept d'étant est le plus fondamental, le premier à s' imprimer dans l' esprit; à ce titre, il est antérieur au concept de vrai, par exemple.

4 Jean Duns Scot, Quaestiones in librum Porphyrii Isagoge, q. 34, $\mathrm{n}^{\circ}$ 1, p. 213. Pour un aperçu des interpretations dans la scolastique latine, on consultera Porro 2014. 
ces propositions laisseraient Duns Scot indifférent, mais en tout état de cause, il ne juge pas opportun d'éclairer ces questions par des références au De causis. En fait il n'y a que deux propositions qui retiennent son attention et qui accaparent la majorité des références.

\section{La thèse sur la réduction à l'unité}

Tout d' abord, la proposition selon laquelle toute multiplicité doit être ramenée à l'unité. En fait, telle quelle, cette thèse appartient plutôt au corpus de l'Elementatio theologica de Proclus ${ }^{5}$, mais il est possible de la relier à un morceau de la proposition XXXI(XXXII) du De causis: «De là vient qu'il y a unité pour l'Un pur et vrai et aussi pour les autres uns et qu'il n'y a d'unité que par l'Un vrai qui est cause de l'unité ${ }^{6}$, et Duns Scot lui-même la relie explicitement au Liber de causis dans son Commentaire aux Catégories ${ }^{7}$. Il cite cette proposition à plusieurs reprises, en particulier dans les Theoremata ${ }^{8}$ dans les Questions sur la Métaphysique ${ }^{9}$, dans l'Ordinatio ${ }^{10}$ : «Toute pluralité doit être ramenée à l'unité, au moins dans la mesure du possible. »

Dans les Questions sur la Métaphysique, la proposition du Liber est présentée comme un argument à l' appui de la thèse de Thomas d'Aquin sur l'unicité de la forme substantielle. Comme on le sait, cette thèse n'a pas la faveur de Scot qui se situe, conformément à l'école franciscaine, plutôt du côté de la pluralité des formes. On remarque ainsi un trait significatif du contexte dans lequel Duns Scot fait recours au De causis. Les thèses de ce traité philosophiques apparaissent souvent dans des discussions théologiques ou des discussions philosophiques ayant un impact réel sur des questions de foi et les propositions du $D e$ causis sont utilisées comme arguments en faveur des thèses erronées aux yeux

5 Cf. Proclus, Elementatio theologica, c. 21, p. 273: Omnis ordinis multitudo ad unam reducitur unitatem.

6 Liber de causis, $\mathrm{xxxI}(\mathrm{XXX1})$, n. 218, p. 114: Provenit ergo inde, ut Uni puro et vero et reliquis unis sit unitas iterum, et non sit unitas nisi propter Unum verum quod est causa unitatis (Magnard et alii, p. 85).

7 Jean Duns Scot, Quaestiones super Predicamenta Aristotelis, q. 4 n ${ }^{\circ}$ 8, p. 274: Item omnis multitudo est reducibilis ad unitatem, per Auctorem De Causis.

8 Jean Duns Scot, Theoremata, Pars 1, B, prop. $5, \mathrm{n}^{\circ} 35, \mathrm{p} .6 \mathrm{o}$.

9 Jean Duns Scot, Quaestiones in Metaphysicam, viI, q. 20, n. 26, p. 385: Unitas videtur ponenda quantum est possible.

10 Jean Duns Scot, Ordinatio I, d. 2, pars 2, q. 1-4, n. 301, p. 305: Omnis pluralitas reducitur ad unitatem vel ad paucitatem tantam ad quantam reducere potest; ergo pluralitas principiorum activorum reducetur ad unitatem vel ad tantam paucitatem ad quantam potest reduci. 
de Duns Scot. Il ne s' agit là que d'une tendance, ce n' est vrai qu'ôs epi to polu (la plupart du temps) comme dirait Aristote. Le Livre des causes semble constituer pour notre théologien un indicateur utile bien que pas toujours tout à fait fiable de ce à quoi la raison humaine et donc la philosophie peuvent parvenir par leurs propres forces. Il est donc toujours intéressant de garder ses thèses à l'esprit, même si, pour Duns Scot, l'esprit humain dispose de la révélation qui lui permet de corriger certaines thèses erronées de la philosophie et de voir plus loin que celle-ci'11.

Dans certaines occurrences, l'autorité du De causis est vue positivement parce qu'il permet de nuancer ou de corriger d'autres thèses philosophiques incorrectes pour lesquelles il n' est donc pas nécessaire de recourir à des arguments révélés, puisque ces thèses peuvent être corrigées par une discussion philosophique menée selon la droite raison.

\section{La causalité supérieure de la cause première}

L'autre thèse du De causis à laquelle il est fait massivement allusion - elle concentre à elle seule la grosse majorité des occurrences - est la première thèse, la thèse de la supériorité de la cause première sur la causalité des causes secondes. Tous les lecteurs de Duns Scot savent par expérience qu'il attache une importance considérable à la problématique des causes essentiellement ordonnées, c' est-à-dire à la synergie entre une cause supérieure, équivoque et une cause inférieure, univoque, thème philosophique majeur déjà bien antérieurement à lui, mais dont il étend le champ d'application à des questions nouvelles comme celle de l' acte de connaissance, des rapports entre intelligence et volonté, etc. Derrière ce modèle omniprésent des causes essentiellement ordonnées, il y a toujours, bien entendu, en arrière-fond la présence implicite de la proposition I du De causis. Donnons quelques exemples de ces références à la première proposition.

Au livre des Questions sur la Métaphysique II q. 3, Duns Scot aborde une question capitale: l'âme unie au corps peut-elle dans notre état connaître les quiddités des substances séparées? Et pour la traiter il aborde la célèbre thèse de Thomas d'Aquin selon laquelle, en cette vie, il nous est impossible de parvenir à une connaissance intuitive de ces intelligences, l'esprit humain en étant réduit à tirer les intelligibles des phantasmes. A l'appui de la thèse de

11 A ce sujet, cf. infra la discussion sur la place des Idées dans la génération univoque. 
Thomas, Scot allègue un argument relativement original à mes yeux tiré de la première position du De causis. Il est établi qu' en cette vie nous ne pouvons connaître la quidddité divine. Mais Dieu est la cause première et, en vertu de la proposition I du De causis, sa causalité est supérieure à celle des causes inférieures et donc à celle des intelligences séparées. Dieu est donc plus connaissable à partir de ses effets que les causes secondes. Si, tout en étant plus connaissable par nous, la quiddité divine demeure néanmoins inconnue, a fortiori celle des intelligences séparées inférieures le sera-t-elle aussi. En conséquence, la connaissance de ces quiddités purement immatérielles ne nous est pas accessible en cette vie. En clair ces substances séparées ne nous sont pas sensibles ${ }^{12}$.

Pour Thomas - en tout cas pour la plupart de ses textes sur la question -, l'homme en est réduit en cette vie, comme premier objet de son intellect, à la quiddité de la chose sensible. Ce n'est qu' après la mort que l'âme séparée pourra intuitionner les substances séparées. Duns Scot n' est pas d' accord avec cette thèse; l'intellect humain est par nature ouvert sur la totalité de l'étant, et le fait qu'il soit incarné ne change rien quant à sa nature ni à ses capacités intrinsèques. Il reprend à cet égard la thèse d'Avicenne, avec cette différence que, selon lui, il ne s' agit pas d'une thèse purement philosophique: Avicenne a subi là l' influence de la révélation coranique et de ses promesses concernant l'au-delà. De fait, nous sommes bien obligés de constater que nous sommes restreints en tant qu'homo viator aux quiddités des choses sensibles, mais ce n' est pas dû pour Duns Scot au statut incarné de notre intellect, mais plutôt aux suites du péché originel, semble-t-il. Nous retrouvons ici l' usage déjà repéré dans le cas précédent: la thèse du Liber vient à l' appui d' une thèse qui n' est pas fausse en soi: nous sommes effectivement dans notre état actuel réduits aux quiddités des choses sensibles, mais qui n' en est pas moins insuffisante dans sa compréhension de ce qui est en jeu et qui n' est pas compatible sur certains points avec la révélation. Montrer qu' ici encore Thomas concède trop aux philosophes étant un objectif de surcroît, mais annexe.

12 Jean Duns Scot, Quaestiones in Metaphysicam, II, q. 2-3, $\mathrm{n}^{\circ}$ 24, p. 207: Tum quia effectus, quinon adaequat potentiam causae, non ducit in cognitionem quiditatis causae; substantiae separatae, si cognoscantur per illa quae faciunt phantasmata in nobis, tantum cognoscuntur per huiudmodi effectus, scilicet non adaequantes causam, ergo etc. Prima propositione patet, quia quiditas talis causae excedit talem effectum. Tum quia finiti ad infinitum nulla est proportio, ergo nec effectus alicuius sensibilis ad quiditatem Dei. Si ergo quiditas Dei, qui est prima causa effectus non posit cognosci per effectum, multo magis nec quiditas alicuius substantiae separatae, cum quaelibet alia causa minus causet quam prima, secundum primam propositionem De Causis. 
Donnons encore un troisième exemple tiré des Questions sur la Métaphysique $^{13}$ : dans une discussion portant sur la question de savoir si Aristote avait raison d' affirmer que pour expliquer le processus de la génération univoque, les Idées de Platon ne sont pas nécessaires, Duns Scot rapporte la thèse d' Averroès selon laquelle la thèse d'Aristote est correcte et il relaie également un argument d'Averroès: toute cause produit un effet semblable à elle; si une Idée était cause, elle produirait par conséquent une forme pure et non un composé matière-forme. Duns Scot n'est pas d'accord avec cet argument: il n' est pas toujours vrai qu'une cause produise nécessairement quelque chose de semblable à elle: la preuve en est que «ce que peut la cause inférieure, la cause supérieure le peut aussi »; dès lors une cause immatérielle et en particulier la cause première peuvent produire des substances matérielles, c' est-à-dire des composés matière-forme. Lorsqu'une cause supérieure agit volontairement, elle peut restreindre sa puissance et produire des effets très dissemblables de sa perfection propre. C'est ce que résulte en particulier pour la question de la création: Dieu est susceptible de produire à lui seul ex nihilo une multitude de composés matière-forme qui sont autant d' effets très éloignés de lui.

La thèse d'Aristote est néanmoins correcte sur le fond et Averroès a raison de la soutenir mais il le fait avec une argumentation en partie erronée. La référence au principe «tout ce que peut la cause inférieure, la cause supérieure le fait aussi », permet donc de rectifier ce qu' il y avait de défectueux dans la position d'Averroès. Cet argument évoque naturellement la première proposition du Liber de causis et l'éditeur considère du reste explicitement qu'il s' agit là d'une référence à cet ouvrage, même si Scot ne le dit pas explicitement. On peut d'ailleurs à mon avis discuter la question de savoir si ce principe «tout ce que peut la cause inférieure, la cause supérieure le peut aussi » découle bien de la proposition I. Celle-ci affirme en fait que «tout ce que pose la cause seconde, la cause première le pose aussi, mais sous un mode plus noble, plus relevé», ce qui n' est pas tout à fait la même chose. Mais il est clair que le principe renvoie en tout cas à des problématiques très proches de celles du De causis et Duns Scot révèle en définitive un instinct philosophique particulièrement sûr en critiquant Averroès à partir d'arguments néoplatoniciens comme ceux du Livre des causes.

13 Jean Duns Scot, Quaestiones in Metaphysicam, viI, q. 11, $\mathrm{n}^{\circ}$ 12-13, p. 184: Contra:quod prima ratio non probet consequentiam: 'Omne agens, quantum est de se, producit sibi simile'. Instantia: actio primae causae minus dependet a materia quam actio alicuius ideae, si esset. Sed Primum non potest producere sibi simile semper, sed producit meteriam sicut formam et similiter compositum. Quia 'quidquid potest causa inferior, potest et superior', sed inferior potest generare compositum; igitur etc. 
Nous retrouvons cet adage «la cause première cause plus que la cause seconde» dans des discussions plus spécifiquement théologiques concernant la vie trinitaire. Dans une question de l'Ordinatio consacrée à la spiration du Saint-Esprit: Est-ce que le Père et le Fils spirent d' égale façon le Saint-Esprit? en d'autres termes: le Père et le Fils produisent-ils à égalité la personne du Saint-Esprit ou faut-il accorder un rôle plus important au Père dans cette production?, question derrière laquelle se trouve naturellement toute la polémique sur le Filioque, Duns Scot introduit un argument intéressant issu du Liber de causis. La question de fond était tranchée dogmatiquement depuis longtemps dans l'Eglise latine, pour laquelle le Saint-Esprit procède du Père et du Fils comme d'un seul principe ${ }^{14}$. Le principe invoqué par Duns Scot: toute cause première agit et est davantage cause que la cause seconde; la cause première donne l'être, la qualité la plus intime et la plus fondamentale pour l'effet que la causalité des causes secondes viendront simplement déterminer et préciser, mais pas d' une manière aussi intime et profonde et dès lors la causalité de la cause première est la causalité proprement essentielle et le fondement de la causalité des autres causes; il est intéressant d' observer que la référence à la proposition I du De causis est renforcée par l' autorité d' Aristote. Duns Scot cite un texte de la Métaphysique et des Seconds Analytiques: «Les principes des choses nécessaires sont toujours les plus vrais, car ils sont causes de la vérité pour les autres choses $»^{15}$. À suivre ces arguments, le Père est donc davantage principe de la procession du Saint-Esprit que le Fils à cause de son statut de cause première dans la production des personnes. De nouveau, le texte du $D e$ causis est mis au service d' une thèse contraire à la vraie foi. Duns Scot répondra que l' argument du Liber vaut pour les causes essentiellement ordonnées; ces causes se caractérisent par des vertus causales différentes, avec une vertu causale plus éminente et plus importante dans le chef de la cause supérieure. Dans ce cas effectivement la cause première cause plus et différemment des causes secondes. Mais ce n' est pas le cas ici, où il n' est pas question de causes, mais de principes ${ }^{16}$. Le Père communique au Fils toute sa vertu principiante, de sorte

14 Aurelius Augustinus, De Trinitate, v, c. 14 n. 15: Pater et Filius sunt unum principium ad Spiritum Sanctum, sicut Pater et Filius et Spiritus Sanctus sunt unum principium a creaturam.

15 Jean Duns Scot, Ordinatio I, d. 12, q. 2, $\mathrm{n}^{\circ} 58$, p. 15: 'Omnis causa prima plus agit quam secunda', ex prima propositione De Causis; et patet ex ficto Philosophi I Posteriorum et II Metaphysicae: 'Necessariorum principia semper esse verissima necesse est, quia sunt aliis causa veritatis.' Ergo omne prius producens, plus producit quam secundum producens. Pater videtur prius producere, quia dat Filio producere, ergo.

16 Jean Duns Scot, Ordinatio I, d. 12, q. 2, n 68, p. 63: Illa propositio vera est de causa et causato, propter hoc quos in causis ordinatis est alia virtus causandi et principalior in priore, non 
que le Père et le Fils spirent l'Esprit-Saint comme un seul principe et une seule vertu principiante, dans une égalité complète, même si cette vertu principiante est «par soi » dans la personne du Père et «reçue en totalité» dans la personne du Fils.

On retrouve la même référence au De causis dans une discussion très similaire à la précédente: les trois personnes divines sont-elles d' égale puissance? Il semble qu'il faille l'affirmer sinon peut-on véritablement parler de Trinité? Néanmoins le Père peut engendrer un Fils alors que le Fils ne peut naturellement pas le faire. Doit-on dès lors parler d'égale puissance pour ces deux personnes? Parmi les objections à une égalité de puissance, nous retrouvons la proposition I du De causis, «la cause première cause davantage qu'une cause seconde », et elle est appuyée par ls mêmes références à Aristote: Métaphysique II et Seconds Analytiques $\mathrm{I}^{17}$. Cette objection reçoit une réponse qui va tout à fait dans le même sens que celle fournie pour la spiration du Saint-Esprit et Scot renvoie d' ailleurs explicitement à ce qu'il avait donné comme réponse à ce sujet ${ }^{18}$.

On a la nette impression que, pour le Docteur Subtil, la doctrine du De causis n' est pas pertinente pour sonder la vie intime même de Dieu. Alors que dans le cas d' une cause, son effet est extérieur à elle et par là inévitablement d'un rang inférieur à celle-ci, dans le cas d'un principe auquel le principié est immanent, le principié peut et même doit être d'égale perfection à celle de son principe. Le De causis aurait tendance à mener à des conclusions qui menacent une compréhension correcte du dogme trinitaire. Cette impression se confirme à propos de quelques problématiques touchant les anges. Le De causis est en effet invoqué à propos de plusieurs questions les concernant. Ainsi, dans le traitement de la question: l' ange a-t-il besoin de raisons distinctes de connaissance pour connaître distinctement les quiddités créées $^{19}$ ? (en clair, a-t-il besoin

autem in principio quod non est causa, quia ibi non est alia virtus principiandi. Ita est in proposito, et ideo non principiat primum principians quam secundum, sicut nec causa superior plus causeret si eadem virtute causativa causaret cum causa secunda, quam causa secunda causaret.

17 Jean Duns Scot, Ordinatio I, d. 20, q. unica, $\mathrm{n}^{\circ}$ 6: Prima causa plus causat quam secunda, secundum auctorem De Causis propositione prima et II Metaphysicae et I Posteriorum; sed Pater dat Filio virtutem causandi, non e converso; ergo Pater plus potest.

18 Jean Duns Scot, Ordinatio I, d. 20, q. unica, $\mathrm{n}^{\circ} 41$ : De causa prima et secunda, responsum est distinctione 12, quod illa propositio veritatem habet propter aliam virtutem sive vim causandi in causa priore et posteriore et illa quae est prioris, est principalior: hoc autem fallit in principio prioris secundum originem et posteroris, in quibus est vis eadem vel virtutis causandi respectu tertiii, cuiusmodi vis est in proposito et ideo in proposito non valet.

Jean Duns Scot, Ordinatio II, d. 3, pars 2, q. $3 \mathrm{n}^{\circ} 353$, p. 569 . 
d'espèces intelligibles distinctes pour connaître distinctement une multiplicité de choses?), le De causis est sollicité en faveur du non. Une cause supérieure cause plus que les causes inférieures ${ }^{20}$ et contient donc en elle plus parfaitement les raisons de ces effets que les causes inférieures. Dès lors, l' ange qui intervient à titre de cause supérieure dans une série de phénomènes contiendra en lui, sous une forme plus parfaite, la quiddité des effets. Il n' est donc pas nécessairement besoin d' une pluralité de raisons pour connaître distinctement des réalités multiples. Mais cet argument ne sera pas retenu sans qu'il y soit répondu directement, ce qui tend à montrer que l'argument du De causis est une interpolation tardive, comme le considèrent les éditeurs. On peut toutefois adresser à cette objection la réponse que $S c o t$ formule à propos d'une objection antérieure: une cause ne contient pas nécessairement sous une forme distincte la quiddité de tous les effets dont elle est capable. Ce n' est vrai en toute rigueur que de la cause première, laquelle est la seule cause complète au sens précis du terme; les autres causes ne sont pas en effet des causes complètes et totales, puisqu' elles requièrent toujours la synergie des causes supérieures (et, selon les philosophes, même des causes inférieures), ce qui induit comme conséquence que ces causes ne contiennent pas nécessairement sous une forme distincte la quiddité de leurs effets.

La discussion sur ce thème a comme interlocuteurs principaux Henri de Gand et Thomas d'Aquin, dont les thèses allaient dans un autre sens: les anges peuvent appréhender distinctement une diversité de contenus sous une seule ratio cognoscendi ou une seule espèce intelligible. Henri, par exemple, attribuait aux anges un habitus intellectuel unique par lequel ils connaîtraient toutes les quiddités. Quant à Thomas d'Aquin, il insistait, dans sa conception de la connaissance angélique, sur le fait que là où des anges inférieurs ont besoin de plusieurs espèces pour appréhender de multiples contenus, un ange supérieur saisit ces mêmes contenus avec beaucoup moins d'espèces. Si l'on suit Thomas, on en arrive pour les anges les plus élevés à une connaissance de très nombreux objets sous quelques espèces très générales, avec le cas-limite de Dieu où tout est connu à travers le prisme de l'unique essence. Thomas est d'ailleurs à ce sujet dans la droite ligne d'une autre proposition du De causis, la proposition $\mathrm{IX}(\mathrm{x})$ où nous lisons ceci: «toute intelligence est pleine de formes; pourtant parmi les intelligences, il y en a qui contiennent des formes moins universelles et d' autres qui contiennent des formes plus universelle ${ }^{21}{ }^{2}$.

20 Jean Duns Scot, Ordinatio II, d. 3, pars 2, q. 3 n $^{\circ} 353$, p. 570 : Item prima propositione De Causis: 'Omnis causa prima plus influit in causatum quam causa secunda'; si igitur angelus sit aliqua causa rei inferoris, continebit ipsam perfecte. 
Mais en réalité, pour Scot, connaître une variété de contenus intelligibles par différentes raisons distinctes est une perfection pour tout intellect, aussi bien pour l' intellect suprême (c' est le sens d' ailleurs pour Scot de la problématique des Idées divines) que pour l'intellect angélique et le nôtre. Ceci est un point important de la conception scotiste que l'intellect divin ne fonctionne pas d'une manière radicalement différente de l' intellect des créatures, en particulier de l'intellect humain ${ }^{22}$.

\section{Le statut angélique et la proposition $\mathrm{xxx}(\mathrm{XXXI})$}

Une autre discussion importante à propos des anges, où le De causis joue un rôle non négligeable concerne la mesure des opérations angéliques par le temps: les opérations angéliques sont-elles mesurées par l'aevum, compris comme perpétuité, ou par le temps?

Intervient alors un argument tiré de la proposition $\operatorname{xxx}(\mathrm{XxxI})$ du De causis: «Entre une chose dont la substance et l' activité sont dans le moment de l'éternité et une chose dont la substance et l'activité sont dans le moment du temps, il existe un intermédiaire: celui-ci est ce dont la substance relève du moment de l'éternité et dont l'opération relève du moment du temps ${ }^{23}$ ». L'ange en effet, selon cet argument, relèverait de cette catégorie d'être, dans la mesure où il serait intermédiaire entre des êtres complètement séparés (des êtres qui, dans leur essence même et leur activité, seraient hors du temps, dans l'éternité) et le monde des corps, immergés dans le temps, tant au niveau de leur substance que de leur activité. Cette assimilation de l'ange à l'âme telle que l' envisageait le De causis est digne d' intérêt mais suscite une certaine perplexité; l'âme est en effet liée constitutivement à un corps dont elle est l'âme et dans la mesure où son activité est orientée vers l' animation du corps qui est le sien, un corps plongé dans le temps par définition, il serait possible d' admettre

tiis sunt quae continent formas minus universales et ex eis sunt quae continent formas plus universales.

22 Jean Duns Scot, Ordinatio II, d. 3, pars 2, q. 3, n 354 , p. 571: Intellectus divinus - secundum multos - plura intelligit distincte per multas distinctas rationes (et propter hoc ponitur ab aliquibus necessitas idearum), et intellectus noster plura praecise intelligit per plures rationes; ergo hoc quod est 'habere plures rationes respectu plurium intelligibilium, nec est ex imperfectione intellectus (quia convenit supremo), nec ex perfectone intellectus (quia convenit infimo); ergo est ex absoluta perfectione intellectus in se.

23 Jean Duns Scot, Ordinatio II, d. 2, pars 1, q. 4, $\mathrm{n}^{\circ}$ 144: Inter rem cuius substantia et actio mensurantur tempore, et rem cuius substantia et actio mensurantur aeternitate, est res media, cuius substantia mensuratur aeternitate (vel aevo) et cuius actio mensuratur tempore. 
que l' activité de l' âme est mesurée par le temps continu. Mais il n'y a pas de raison de considérer qu'il en va de même pour l' ange: il est certes susceptible de poser des opérations à l'égard de corps, et par là d'être lui aussi en relation avec le temps, mais il s' agit là d'opérations extrinsèques et non d' opérations intrinsèques comme la pensée ou la volition qui sont également prises en considération ici. Henri de Gand ${ }^{24}$ considérait aussi, et d' ailleurs Thomas d' Aquin ${ }^{25}$ avant lui également, que les opérations angéliques étaient mesurées par un temps discret. Duns Scot souligne d' ailleurs le caractère somme toute déplacé de cet argument: «il pose que l' ange est vraiment dans le moment du temps non par son activité intrinsèque parce que pour cela il ne poserait ni temporalité ni succession, mais par une activité extrinsèque, à l'égard d'un corps ${ }^{26}$ ». Mais le point le plus significatif de ce passage est ce que Duns Scot ajoute comme appréciation sur le Liber de causis en général ${ }^{27}$ : la doctrine de ce livre est tirée en fait d'Avicenne et cette doctrine est erronée car elle fait des intelligences cosmiques des dieux dont certaines activités seraient mesurées par le temps ${ }^{28}$.

Habituellement le XIII ${ }^{\mathrm{e}}$ siècle attribue la paternité du Liber de causis à Aristote (souvent pour le texte même des propositions) et à Al-Fārābī et Avicenne pour le commentaire, même si Al-Fārābī, il faut bien le dire n' est pour beaucoup de médiévaux qu'un nom. Duns Scot exprime ici comment il perçoit les choses: la doctrine du De causis provient d'Avicenne et c'est cette provenance qui empêche pour ainsi dire de prendre réellement en compte ses affirmations car elle est gâtée par certaines erreurs de la doctrine avicennienne. En fait, quand nous disons que pour Duns Scot, la doctrine du Liber provient d'Avicenne, il nous faut être plus précis. Duns Scot dit précisément: la doctrine du De causis est transmise (ou livrée) selon la doctrine d'Avicenne. La manière la plus simple de comprendre cette expression est de présumer que Scot fait ici une distinction entre la doctrine du De causis proprement dite, qui consisterait en les seules propositions et son interprétation qui désignerait plutôt les textes explicatifs intercalés entre les propositions; c' est elle qui porterait

24 Henri de Gand, Quodlibet XI, q. 11, f. $465^{\mathrm{r}}-467^{\mathrm{v}}$; Quodlibet XII, q. 8, p. 40-46; Quodlibet XIII, q. 7 , p. 43 .

25 Thomas d'Aquin, Summa theologiae, ia, q. 63, a. 7, ad. 4.

26 Jean Duns Scot, Ordinatio II, d. 2, pars 1, q. 4, $\mathrm{n}^{\circ}$ 185, p. 238 : Non quidem intrinsecam operationem (quia ad hoc non poneret potentialitatem neque successionem), sed extrinsecam circa corpus - esse vere in momento temporis.

27 Nous reprenons ici une question que nous avions déjà discutée dans J.-M. Counet 2002.

28 Jean Duns Scot, Ordinatio II, d. 2, pars 1, q. 4, n 185, p. 238: Doctrina De causis tradita est secundum doctrinam Avicennae erroneam, ac si intelligeret ille auctor intelligentias esse quosdam deos et operationes earum mensurari 'nunc' temporis. 
la marque d'Avicenne. Vue sous cet angle, la position de Scot est traditionnelle, en ce qu' elle distingue une double paternité au texte du De causis tel qu' il nous est parvenu, même s'il n'attribue pas explicitement la paternité des thèses à Aristote.

L'interprétation du De causis est erronée sur deux points selon Scot: $1^{\circ}$ les anges sont des créatures et non des dieux; $2^{\circ}$ dans la perspective créationniste, il n' est pas nécessaire qu' ily ait une continuité absolue dans les degrés d' être et qu' ainsi, entre les êtres complètement séparés et les êtres soumis à la matière et au temps, il faille postuler l' existence d'êtres dont la substance est mesurée par l'éternité et l' activité par le temps.

De plus, il n' est pas dit du tout que ce soit là la place de l'ange: on y verrait plutôt celle de l'âme. En fait pour Duns Scot les opérations elles-mêmes des anges ne sont pas mesurées par le temps, mais par l'aevum. Le temps n' intervient qu' à titre purement accidentel dans le cadre des opérations extrinsèques touchant les propriétés des corps (la substance même des corps étant elle aussi mesurée par l'aevum ${ }^{29}$ ).

\section{Henri de Gand et sa réappropriation de l'émanatisme avicennien}

Lorsque Duns Scot a dans son collimateur le De causis et son fondement avicennien il vise également et peut-être encore davantage Henri de Gand et la réinterprétation que celui-ci avait faite de la doctrine du philosophe persan. En effet, s'étant efforcé d' intégrer dans l' ensemble de sa construction philosophique et théologique ce qui pouvait être sauvé de l'émanatisme avicennien, la création est pour le Docteur Solennel l' œuvre de Dieu, c' est-à-dire des trois personnes. Est-ce à dire que les différentes personnes agissent ensemble et de façon indistincte avant l' effectuation ad extra des créatures? Henri pensait que non $^{30}$; il soutenait l'action commune des trois personnes dès qu'il s' agit de

29 Jean Duns Scot, Ordinatio II, d. 2, pars 1, q. 4, n 167, p. 230 : Concedo [...] quod [...] intellectiones angeli mensurantur aevo - et breviter, quaecumque existentia actualis et invariabilis, hoc est cui repugnat ut secundum ipsam sit variatio sive fluxus seu acquisitio partis post partem; nec perpetuitas aliquorum vel corruptio sive annihilatio aliquorum variat mensuram formaliter, dummodo existentia sit eiusdem rationis dum manet. Id., Lectura, II, d. 2, pars 1, q. 4, $\mathrm{n}^{\circ} 16 \mathrm{o}$, p. 151: [...] dico quod esse rei generatae potest comparari ad causam proximam, et ad causam primam a qua potest esse post primum instans. Res non dependet a causa proxima quia est causa rei in fieri (sicut aedificator ipsius domus tantum est causa in fieri); sed Deus est causa rei in esse. Unde esse rei, ut sequitur primum instans, tantum est a Deo dependens, - et sic mensuratur aevo. Cf. Porro 1996, p. 251-256. 
causalité ad extra stricto sensu, mais en ce qui concerne l'élaboration immanente à la Trinité du plan créateur, il pensait que chaque personne avait sa contribution spécifique ${ }^{31}$ : le Père n'a qu'une connaissance théorique de tous les possibles et, parallèlement à cette connaissance, sa volonté se porte vers ces possibles par un simple amour d' inclination. Dans la personne du Fils, les possibles seraient considérés dans une sagesse dispositive et pratique: ils seraient vus comme susceptibles d'être concrètement réalisés. De manière tout à fait parallèle, ils sont voulus dans le Saint-Esprit d'un acte de volonté libre et explicite qui les atteint dans leur particularité32 et leur confère l' existence effective.

A travers la distinction de la connaissance théorique qui considère les réalités qu' elle saisit sous la modalité de l'universel, connaissance théorique qui est l'œuvre du Père, et l'art pratique qui saisit ces mêmes réalités comme particulières et comme devant être réalisées par une action qui ne peut porter que sur le particulier, art qui est l'apanage du Fils et de l'Esprit, Henri propose une

in corp. art., p. 33-40: Philosophi tantum ponebant in Deo intellectum simplicis intelligentiae, qua intelligit se et omnia alia et similiter voluntatem simplicem, qua placent sibi omnia secundum quod sunt bona in sua essentia. Talis autem intelligentia, cum hoc quod est naturalis, naturaliter se habet ad intellecta producenda et secundum unum determinatum modum, et similiter est de voluntate concomitante talem simplicem apprehesionem; et ideo necesse habuerunt ponere quod de necessitate naturae produxit creaturas extra se et secundum optimum modum producendi (scilicet intellectu et voluntate ut natura, non voluntate ut dispositiva et electiva) et in hoc erraverunt quia secundum eos nulla emanatio intriseca praecedit productionem rerum extra. Sed hoc ut dicit philosophorum opinio non valet quia ad hoc quod Deus producat aliquid extra se, non sufficit simplex notitia et simplex amor declarativa et dispositiva fiendorum et amor incentivus seu affectans productionem eorundem; haec autem notitia declarativa vel dispositiva est Verbum et amor affectans est Spiritus Sanctus: igitur productionem rerum praecedit Verbum et Spiritus Sanctus. Sicut artifex creatus in sapientia artis duplicem habet notitiam de artificiato, unam simplici notitia in arte universali, qua intuetur operanda pure speculative, et aliam dispositivam ad opus, qua in arte particulari concepta de arte universali intuetur ordinem productionis suae (et est cognotio practica, sine qua impossibile est artificem in opus procedere), - similiter est ex parte voluntatis, quod artifex duplicem habet amorem artificii, unum simplicem quo placet ei forma artificii, qui non ordinat ad opus, et alium affectantem quo opus fieri desiderat. Consimiliter est ex parte Dei, quod simplici notitia novit omnia simpliciter et absolute, in notitia vero practica novit omnia sicut in arte dispositiva et declarativa eorum ad opus - et una notitia procedit ex alia; et similiter est ex parte voluntatis de amore simplici et affectante.

31 Sur ce point aussi nous reprenons des considérations développées dans Counet 2002, p. 235-241.

32 Jean Duns Scot, Ordinatio II, d. 1, appendix A, p. 6o7: Sed hoc ut dicit philosophorum opinio non valet quia ad hoc quod Deus producat aliquid extra se, non sufficit simplex notitia et simplex amor complacentiae (quae tantum ad notitiam speculativam pertinent) sed requiritur notitia declarativa et dispositiva fiendorum et et amor incentivus seu affectans productionem eorumdem; haec autem notitia declarativa vel dispositiva est Verbum, et amor affectans est Spiritus Sanctus; igitur productionem rerum praecedit Verbum et Spiritus Sanctus. 
théologie trinitaire de l'acte créateur où les hypostases divines interviennent de manière spécifique avec des causalités complémentaires, non interchangeables entre elles. La création du monde est bien un acte médiatisé, mais pas médiatisé par une série d'intelligences recevant et transmettant selon la perfection de leur essence mais par la génération du Fils et la procession du Saint-Esprit, chez qui les possibles sont conçus et voulus pratiquement en tant que créables.

De cette manière, Henri s' efforçait de trouver un cadre approprié à l'émanatisme avicennien au sein même de la vie trinitaire. Alors que pour Avicenne tout le cosmos procède de Dieu de manière nécessaire, dans un flux créateur se déversant sur un certain nombre de niveaux où il se réfléchit en intelligences et donne naissance à autant de cieux matériels animés, avec une perte progressive de sa simplicité, de sa perfection et de sa fécondité, le monde sublunaire étant la dernière étape de ce processus créateur, chez Henri le processus nécessaire s'épuise dans la génération du Fils, la procession du Saint-Esprit et d' un monde de possibles, d' essences reliées les unes aux autres par des ordres essentiels ${ }^{33}$. Nous retrouvons là le fameux être de l' essence des créatures, l' esse essentiae des créatures en tant que pensées par Dieu. Plus concrètement l'esse essentiae est la créature conçue par la sagesse dispositive du Fils et offerte à l' amour électif de l'Esprit, pour, le cas échéant, la faire passer à l' existence effective, en dehors de sa cause divine, dans le monde réel. Cette conception de l' esse essentiae fut l'objet d'une âpre controverse avec Gilles de Rome, sur laquelle nous n'allons pas revenir ici.

De cette manière, Henri donnait donc une certaine caution théologique à l'émanatisme avicennien et à la doctrine du Liber de causis, tout en ménageant le point capital d'une création effective libre et contingente de la part de Dieu. Henri allait donc très loin dans l'appropriation de la doctrine du De causis et il semble évident que lorsque Duns Scot attaque l' autorité du De causis sur les questions théologiques, ce n'est pas seulement à cause des doctrines philosophiques qu'il charrie, mais aussi et peut-être surtout à cause de l'usage qu' en font certains théologiens, en particulier Henri de Gand.

Duns Scot quant à lui récusera ces conceptions accommodatrices et syncrétistes d'Henri. Il prônera une claire distinction des genres entre domaine du

33 Sur cette notion importante pour Duns Scot d'ordre essentiel, reprise à Henri, cf. Theoremata, pars 8, De Causis, p. 661-671 et le De primo principio, I, 2, p. 2: Quamvis entis sint plurimae passiones quarum consideratio valeret ad propositum prosequendum, tamen de ordine essentiali tamquam de medo decundiori orimo prosequatur isto modo: in hoc primo captulo divisiones quatuor ordinis praemittam, ex quibus colligetur quot sunt ordines essentiales. 
créé et vie intradivine comme entre philosophie et théologie. Il entend rester fidèle à la théologie trinitaire classique selon laquelle la création est l'œuvre commune des trois personnes qui œuvrent à égalité ad extra comme une seule cause du processus. Non, les philosophes comme Avicenne n' ont pas pressenti la Trinité en concevant leur création nécessaire à partir de Dieu et leurs conceptions ne sont guère utiles pour penser le dyanamisme intradivin. Pour Duns Scot, s'il n'y avait eu qu' une personne divine, le processus créateur n' eût pas été fondamentalement différent de ce qu' il est sous nos yeux. Non seulement l'avicennisme et la doctrine du De causis ne permettent pas de penser la vie divine, mais ils ne donnent pas non plus une compréhension satisfaisante de la nature, de la connaissance et de l' activité des anges. Ceux-ci ne ressemblent que d'une manière assez lointaine aux Intelligences des philosophes et à trop vouloir s' appuyer sur les conceptions de ces derniers, on tombe dans l' erreur à leur propos.

\section{Conclusions}

Nous pouvons résumer les points saillants de cette contribution comme suit:

1. Le Liber de causis représente pour Duns une autorité à prendre en ligne de compte dans la mesure où il reflète bien, sur un certain nombre de points, ce à quoi peut parvenir l'intelligence humaine dans la compréhension de la causalité intramondaine.

2. Il doit être considéré comme une reprise synthétique de la pensée d'Avicenne dont il partage les points forts et les faiblesses. Avicenne a pris certaines thèses connues uniquement par la révélation comme étant accessibles à la simple raison, et il a aussi couvert de son autorité des reliquats du polythéisme grec, en particulier dans sa conception des intelligences et des astres. Il s'agit de comprendre correctement l'extension de son autorité et ne pas l'étendre indûment. Cela vaut aussi pour le De causis.

3. Maurice du Port avait dit que Scot suivait en tout Avicenne en philosophie $^{34}$ tant qu' il n'était pas contraire à la foi. Effectivement, sur une série de points concernant la vie trinitaire et la condition angélique, le De causis ne peut se prévaloir d'une véritable autorité.

4. Dans le contexte trinitaire ou angélique, les références explicites au Liber sont souvent des arguments étayant des thèses contraires à la foi, ou des

34 Annotationes in Metaphysicam Scoti, IV, cité par Gilson 1927, p. 148-149: Favet namque Avicennae inter philosophos ubique, nisi sit contra fidem [...]. 
thèses philosophiques ayant des conséquences fâcheuses pour cette dernière. Mais en philosophie, on rencontre aussi un usage redresseur du Liber: il aide à retrouver la vérité philosophique, en mettant en évidence des déformations de celle-ci.

5. Enfin, Henri de Gand, et dans une certaine mesure également Thomas d'Aquin, se sont fourvoyés en accordant trop au De causis. Ils ont par là contribué à fausser le juste rapport entre philosophie et théologie en défendant des thèses mettant en danger l' intégrité de la foi, que ce soit sur le plan directement théologique chez Henri avec la doctrine de la création ou sur le plan philosophique chez Thomas, avec la théorie de l'unicité de la forme substantielle ou sa conception sur le premier objet de l' intellect. Il resterait à voir, mais c'est là quelque chose qui dépasse le cadre de cette contribution, dans quelle mesure il serait correct de dire que le célèbre traité De primo principio où Duns Scot, pour reprendre encore une fois Etienne Gilson, reprend l' argument du Proslogion avec toutes les ressources dialectiques de son temps ${ }^{35}$, ne serait pas une sorte de Liber de causis revu et corrigé par Duns Scot de façon à coller au mieux avec les requêtes de la foi. Le fait que ce traité soit essentiellement un traité sur la métaphysique des causes et qu'au terme de l' argument une cause première unique, infinie, intelligente et dotée de volonté soit déclarée existante en acte, confirmant ainsi rationnellement l'invocation du Nom de Dieu révélé à Moïse ${ }^{36}$ irait clairement dans ce sens, mais il faudrait bien entendu une étude plus approfondie pour mettre en évidence tout le travail scotiste de réappropriation du contenu du De causis.

\section{Bibliographie}

\section{Sources primaires}

Liber de causis - A. Pattin, "Le Liber de causis. Édition établie à l' aide de go manuscrits avec introduction et notes", in Tijdschrift voor Filosofie 28 (1966), p. 90-203. Liber de Causis, trad. française: P. Magnard, O. Boulnois, B. Pinchard, J.-L. Solère, La Demeure de l'être. Autour d'un anonyme, Paris, Vrin, 1990.

35 Gilson 1952, p. 178.

36 Jean Duns Scot, De primo principio, I, 1, p. 2: Domine deus noster, Moysi servi tuo de tuo nomine filiis Israel proponendo a te doctore verissimo scicitanti, sciens quid posset de te concipere intellectus mortalium, nomen tuum benedictum reserans respondisti: 'Ego sum qui sum. Tu es verum esse, tu es totum esse. Hoc credo, hoc si mihi esset possibile, scire vellem. Adiuva me, domine, inquiread quantam cognitionem de vero esse, quod tu es, possit pertingere nostra ratio naturalis ab ente, quod de te praedicasti, inchoando. 
Augustin, De Trinitate Libri XIV, cura et studio W.J. Mountain auxiliante Fr. Celarie, Turnhout, Typographi Brepols editores Pontificii, MCMLXVIII.

Henri de Gand, Quodlibet vi, éd. G.A. Wilson, Leuven, Leuven University Press, 1987. Henri de Gand, Quodlibet XI, éd. J. Badius, Paris, 1518.

Henri de Gand, Quodlibet XII, q. 1-3o, éd. J. Decorte, Leuven, Leuven University Press, 1987.

Henri de Gand, Quodlibet XIII, éd. J. Decorte, Leuven, Leuven University Press, 1985. Jean Duns Scot, Quaestiones in librum Porphyrii Isagoge, éd. R. Andrews, G. Etzkorn,

G. Gal, R. Green, T. Noone, R. Wood, in Opera Philosophica I, The Franciscan Institute, St Bonaventure University, St Bonaventure New York, 1999.

Jean Duns Scot, Quaestiones super Predicamenta Aristotelis, Opera Philosophica I, éd. R. Andrews, G. Etzkorn, G. Gal, R. Green, T. Noone, R. Wood, The Franciscan Institute, St Bonaventure University, St Bonaventure New York, 1999.

Jean Duns Scot, Theoremata, in Opera Philosophica II, éd. M. Dreyer, H. Möhle collaborante G. Krieger, The Franciscan Institute, St Bonaventure University, St Bonaventure New York/ The Catholic University of America, Washington D.C., 2004.

Jean Duns Scot, Quaestiones in Metaphysicam, in Opera Philosophica III-IV, éd. R. Andrews, G. Etzkorn, G. Gal, R. Green, F. Kelley, G. Marcil, T. Noone, R. Wood, The Franciscan Institute, St Bonaventure University, St Bonaventure New York, 1997.

Jean Duns Scot, Abhandlung über das erste Prinzip, hrsg. und übersetzt von W. Kluxen,

Wissenschaftliche Buchgesellschaft, Darmstadt, 1974.

Jean Duns Scot, Traité du Premier Principe, trad. française J.-D. Cavigioli, J.-M. Meilland,

Fr.X. Putallaz sous la direction de R. Imbach, Genève, Lausanne, Neuchâtel, 1983.

Jean Duns Scot, Ordinatio I, in Opera Omnia 3, éd. C. Balić, M. Bodewig, S. Bušelić, P. Čapkun-Delić, B. Hechich, I. Jurić, B. Korošak, L. Modrić, I. Montalverne, S. Nanni, B. Pergamo, F. Prezioso, I. Reinhold, O. Schäfer, Vatican, Typis Polyglottis Vaticanis, 1954.

Jean Duns Scot, Ordinatio II, in Opera omnia 7, éd. C. Balić, C. Barbarić, S. Bušelić, B. Hechich, L. Modrić, S. Nanni, R. Rosini, S. Ruiz de Loizaga, C. Saco Alarcón, Vatican, Typis Polyglottis Vaticanis, 1973.

Proclus, Elementatio theologica, transl. a Guillelmo de Morbecca, éd. H. Boese, Leuven, Leuven University Press, 1987.

\section{Sources secondaires}

Counet, J.-M. (2002), «Avicenne et son influence sur la pensée de Duns Scot», dans J. Janssens, D. De Smet (éds), Avicenna and His Heritage, Leuven, Leuven University Press, p. 246-25o.

Gilson, E. (1927), «Avicenne et le point de départ de Duns Scot», dans Archives d'Histoire doctrinale et littéraire du Moyen Age 2, p. 90-148.

Gilson, E. (1952), Jean Duns Scot. Introduction à ses positions fondamentales, Paris, Vrin. 
Porro, P. (1996), Forme e modelli di durata nel pensiero medieval. L'aevum, il tempo, la categoria quando, Leuven, Leuven University Press.

Porro, P. (2014), «The University of Paris in the thirteenth century:Proclus and Liber de causis », in S. Gersh (éd.), Interpreting Proclus. From Antiquity to the Renaissance, Cambridge, Cambridge University Press 2014, p. 264-299. 